\title{
Democratizing Children's Engagement with the Internet of Things through ConnectUs
}

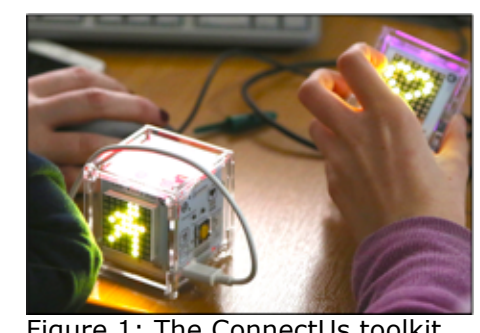
comprising of interactive sensing cubes with Bluetooth connectivity

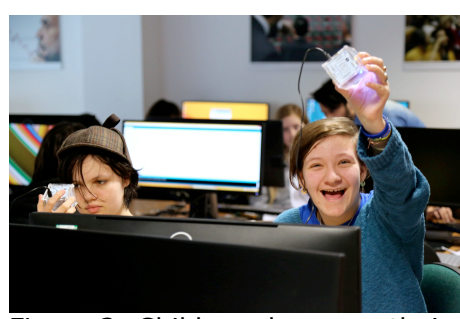

Figure 2: Children showcase their Internet of Things creations at a ConnectUs workshop

\section{Zuzanna Lechelt \\ University College London \\ UCL Interaction Centre \\ London, UK, WC1E 6BT \\ susan.lechelt.15@ucl.ac.uk \\ Venus Shum \\ University College London \\ UCL Interaction Centre \\ London, UK, WC1E 6BT \\ v.shum@ucl.ac.uk}

\section{Yvonne Rogers}

University College London

UCL Interaction Centre

London, UK, WC1E 6BT

y.rogers@ucl.ac.uk

\section{Nicolai Marquardt}

University College London

UCL Interaction Centre

London, UK, WC1E 6BT

n.marquardt@ucl.ac.uk

Permission to make digital or hard copies of part or all of this work for personal or classroom use is granted without fee provided that copies are not made or distributed for profit or commercial advantage and that

copies bear this notice and the full citation on the first page. Copyrights for third-party components of this work must be honored. For all other uses, contact the Owner/Author.

Copyright is held by the owner/author(s).

Ubicomp/ISWC'16 Adjunct, September 12-16, 2016 Heidelberg, Germany ACM 978-1-4503-4462-3/16/09.

http://dx.doi.org/10.1145/2968219.2971435

\begin{abstract}
The emerging Internet of Things (IoT), through which billions of everyday objects are becoming embedded with the abilities to sense their environment, compute data, and wirelessly connect to other devices, has been widely recognized as the new disruptive technology of our time. The predicted ubiquity of connected devices indicates that IoT technologies are quickly becoming an important part of the digital fluency curriculum, however, no research yet exists on suitable pedagogical approaches for teaching children about the IoT. The current research explores the design and deployment of a pedagogical approach and associated tangible toolkit, ConnectUs, that will enable 10-13 year old children to explore and design for the Internet of Things.
\end{abstract}

\section{Author Keywords}

Tangible interfaces; ubiquitous computing; playful learning; Internet of Things; ConnectUs; SenseMe; CodeMe.

\section{ACM Classification Keywords}

H.5.m. Information interfaces and presentation (e.g., HCI): Miscellaneous; K.3.1 Computers and Education: Computer Uses in Education Computer-assisted instruction; H.5.2 Information Interfaces and Presentation: User Interfaces - User-centered design 


\section{Introduction}

In recent years, computing curricula worldwide have rapidly begun to shift in focus, moving away from teaching children how to consume digital products, and toward enabling creation with technology. From the recent redesign of the UK national computing curriculum, which now places high emphasis on tinkering and making [10], to the emergence of the international maker movement [6], children worldwide have increased opportunities to learn about computing by engaging with technology critically and creatively. This is a promising trend, enabled by years of research on best practices for lowering the entry threshold to programming (e.g., $[7,8]$ ) and electronics (e.g., [3]).

However, the field of computing is rapidly changing, and along with it, so is the scope of digital fluency. With the rise of ubiquitous computing and particularly the Internet of Things (IoT), it is crucial to expose the next generation of learners to the central topics underlying these technologies. This is a challenging task, given that these topics, which include embedded programming, wireless sensor networks, and distributed systems, are quite complex and normally only taught at the higher education level.

Therefore, this work addresses two questions: 1) How can the complex concepts underlying the IoT be brought down to a level that is accessible to young learners?

2) What interfaces can be designed to lower the entry level for creative exploration of IoT technologies?

This paper describes the conceptual development of an IoT pedagogy for children aged $10-13$, as well as its instantiation through ConnectUs (Figure 1), a novel tangible toolkit for teaching IoT concepts.

\section{Conceptual Development}

Within this research, the first subject of interest is to clarify what topics are fundamental to understanding the IoT, and how they can be brought down to a leve that is accessible to children. To this end, the IoT can be discussed in terms of its two subcomponents:

"things" and the "Internet".

Understanding the IoT crucially involves understanding the physical objects that comprise its basis. To this end, within an IoT pedagogy, it is important for children to understand the functionality of sensors, processors and output devices. Active exploration of these topics is already accessible to children thanks to the rich and varied bodies of work on computational toolkits for making and child-friendly programming languages (e.g., [3]).

However, enabling the active exploration of the "Internet" aspect of the IoT, which relates to networking, connectivity and distributed systems, poses a greater challenge. Although some work has with success explored teaching children about networks and protocols by asking them to physically connect devices to create a networked system [5] within an IoT pedagogy, the goal is rather to explore the situated use of networks within IoT systems. For example, how do networked sensors distributed around a room communicate to a central device, how does this device then act on this sensor data, and in what cases can this system break down?

To this end, we argue that a good starting point is not to teach about networking and distributed systems per se, but rather about systems thinking concepts. Core 



Figure 3: Sketches of the ConnectUs cube showcasing its features. These include various sensors, an Arduino

microcontroller, an LED matrix and Bluetooth. systems thinking concepts, including interdependence, feedback loops and emergent behaviours in a system (e.g., [9]), can be seen as conceptual predecessors to more technically advanced aspects of the IoT like networking protocols and distributed systems. As such, learning about these concepts will provide computing novices with a basis to further explore and think about IoT technologies.

\section{The ConnectUs Toolkit}

To instantiate an approach for teaching children about the IoT topics described above, we have developed a novel tangible toolkit, which we call ConnectUs. ConnectUs consists of interactive cubes embedded with a variety of sensors, output devices, and Bluetooth technology (Figure 3). The cubes, which include an Arduino [2] microcontroller, can be explored and programmed by children individually to enable learning about the hardware aspects of the IoT. Additionally, by wirelessly connecting many cubes together via Bluetooth, it is possible to extend the variety of concepts taught to include those related to networking and connectivity, such as the systems thinking principles described above. The toolkit is designed to be discovery-based, social and to encourage creative crafting and tinkering.

\section{Learning with ConnectUs}

Based on the ideas described above, we are developing a two-stage interactive workshop series with the ConnectUs toolkit to introduce children to the IoT topics of hardware and networked systems. On the basis of theoretically motivated principles from the learning sciences (e.g., [7]), as a means of scaffolding learning, in each workshop children alternate between exploring IoT functionality through pre-programmed activities on the ConnectUs toolkit, and creatively constructing their own IoT devices and systems by reprogramming the toolkit in the Arduino IDE [2], with the assistance of adult helpers and step by step worksheets. All learning activities within the workshops have gone through several stages of iterative design, and have been evaluated for their potential to convey the target learning outcomes.

A total of 170 10-12 year old children participated in the hardware workshop. Within the workshop, children first explored pre-programmed sensor-output transforms, and were asked to collaboratively discuss how they thought their functionality was practically implemented. For example, in one of the transforms, the accelerometer embedded in the cube mapped different speeds of shaking onto different colours of the embedded neopixel light. After exploring several such transforms, children were given the opportunity to reprogram the cube in Arduino [2] in order to customize their own sensor-output mappings. At this stage, children could, for example, create a "night light", which turned on the embedded neopixel light when the light sensor detected that the light level in the room was below a certain threshold.

While more comprehensive evaluation of the deployment of this workshop is underway, we have observed that following the workshop, many children were able to robustly describe the functionality of sensors and output devices, indicating a deep understanding of the target learning outcomes. Additionally, evaluations of satisfaction surveys showed that the workshop was very positively received by children, and preliminary analysis of videos of the workshop indicated that it was conducive to fostering 
high levels of engagement and sustained collaboration between peers.

The networked systems workshop, which is in earlier stages of development, will follow the same structure as the hardware workshop. Children will first explore and discuss pre-programmed activities incorporating Bluetooth connectivity, which are aimed at conveying concepts related to systems thinking. For example, to convey the idea of interdependence between parts in a system, shaking one ConnectUs cube at a time turns on a red light in the cube, while shaking two simultaneously turns on a purple light in both cubes.

For the expressive stage of the workshop, we are in the process of developing an easy to use end-user programming interface for the ConnectUs toolkit, which will enable children to "program" networked sensoroutput functionality between multiple cubes. For example, children could collaboratively create an "earthquake detector system" by monitoring the accelerometers embedded in all cubes, and displaying a flashing warning light on the LED matrix of all cubes when one of the cubes detects sufficient acceleration. This end-user programming interface will aim to facilitate creative exploration of systems thinking principles like interdependence and emergent behaviours, allowing children to gain a situated knowledge of the IoT.

\section{Conclusion}

Drawing inspiration from the learning sciences and the maker movement, we have developed an interface that will enable young computing novices to explore and creatively engage with core topics underlying IoT. While our approach is still a work in progress, preliminary evaluations of deployments of ConnectUs with children have shown that the interface has much potential for lowering the entry threshold for exploring IoT topics.

\section{References}

1. Farha Ali. 2015. Teaching The Internet of Things Concepts. In Proceedings of the WESE'15: Workshop on Embedded and Cyber-Physical Systems Education. ACM, 2015.

2. Arduino. Arduino. Retrieved June 1, 2016 from https://www.arduino.cc

3. Paulo Blikstein. 2015. Computationally Enhanced Toolkits for Children: Historical Review and a Framework for Future Design. In Interaction 9.1: 1-68

4. CodeMe. The CodeMe Toolkit. Retrieved June 1 , 2016 from http://www.codeme.io.

5. Yvon Feaster et al. 2014. Serious toys II: teaching networks, protocols, and algorithms. In Porceedings of the 18th ACM conference on Innovation and technology in computer science education. ACM

6. Erica R. Halverson and Kimberly Sheridan. 2014. The maker movement in education. In Harvard Educational Review 84.4: 495-504.

7. Seymour Papert. 1980. Mindstorms: Children, computers, and powerful ideas. Basic Books, Inc.

8. Mitchel Resnick, et al. 2009. Scratch: programming for all. In Communications of the ACM 52.11: 6067.

9. Barry Richmond \& Steve Peterson. 2001. An introduction to systems thinking. High Performance Systems, Incorporated.

10. UK Department of Education. 2013. National curriculum in England: computing programmes of study. 